\title{
PREDIÇÃO DA VIDA ÚTIL DE FILÉS DE PEITO DE FRANGO RESFRIADOS EMBALADOS A VÁCUO E COM ATMOSFERA MODIFICADA EM CONDIÇÕES DE ARMAZENAMENTO NÃO ISOTÉRMICO
}

\author{
MARIA ELIZABETH DE PAULA CANÇADO MEZAROBA* \\ DANIEL ANGELO LONGHI** \\ FRANCISCO ENEIAS KONKEL ${ }^{* * *}$ \\ GLÁUCIA MARIA FALCÃO DE ARAGÃO****
}

\begin{abstract}
A temperatura é um fator ambiental que tem grande impacto sobre o crescimento de micro-organismos deteriorantes em alimentos. Em condições reais de armazenamento, transporte e comercialização de produtos cárneos podem ocorrer grandes variações de temperatura, e com isso o estudo do crescimento de bactérias ácido-lácticas (BAL) em condições de armazenamento não isotérmico tem grande importância. $O$ objetivo deste trabalho foi propor e validar modelos matemáticos (Gompertz modificado e Baranyi) para condições não isotérmicas e predizer a vida útil de filé de peito de frango resfriado a partir do crescimento de BAL em embalagens a vácuo e com atmosfera modificada (ATM, 50\% $\mathrm{CO}_{2}$ e $50 \% \mathrm{~N}_{2}$ ). Os resultados apontaram que os modelos de Gompertz modificado e Baranyi foram capazes de predizer o crescimento de BAL em filés de peito de frango embalados a vácuo e com ATM, o que foi verificado através dos índices estatísticos $R^{2}$, MSE, fatores bias e exatidão, sendo que o modelo de Gompertz modificado apresentou as predições mais conservadoras (menores valores) sobre a duração da vida útil. A vida útil observada dos filés de peito de frango foi de aproximadamente 12 e 6 dias nas embalagens com ATM e a vácuo, respectivamente, mostrando que a embalagem com ATM levou ao aumento da vida útil do produto. Além disso, os modelos matemáticos propostos e validados neste estudo podem ser utilizados para predizer a influência de diferentes condições não isotérmicas sobre o crescimento de BAL em filés de peito de frango.
\end{abstract}

PALAVRAS-CHAVE: BACTÉRIAS ÁCIDO-LÁCTICAS; CRESCIMENTO MICROBIANO EM ALIMENTOS; MICROBIOLOGIA PREDITIVA.

\footnotetext{
* Doutoranda em Engenharia de Alimentos, Departamento de Engenharia Química e Engenharia de Alimentos, Universidade Federal de Santa Catarina, Florianópolis - SC, Brasil (e-mail: mmezaroba@gmail.com).

** Doutorando em Engenharia de Alimentos, Departamento de Engenharia Química e Engenharia de Alimentos, Universidade Federal de Santa Catarina, Florianópolis - SC, Brasil (e-mail: ealdaniel@hotmail.com)

*** Mestre em Engenharia de Alimentos, Departamento de Engenharia Química e Engenharia de Alimentos, Universidade Federal de Santa Catarina, Florianópolis - SC, Brasil (e-mail: franciscokonkel@auroraalimentos.com.br).

**** Professora Doutora em Engenharia de Alimentos, Departamento de Engenharia Química e Engenharia de Alimentos, Universidade Federal de Santa Catarina, Florianópolis - SC, Brasil (e-mail: glaucia@enq.ufsc.br).
} 


\section{INTRODUÇÃO}

A deterioração microbiológica de carnes ocorre principalmente pelo crescimento de bactérias, como as bactérias ácido-lácticas (BAL), que produzem metabólitos que causam mudanças indesejáveis na aparência, textura e flavor do alimento, produzindo odores e sabores desagradáveis, além de formar limo na superfície dos produtos (BORCH, KANT-MUEMANSB, BLIXT, 1996; CAYRÉ, VIGNOLO e GARRO, 2003; NYCHAS et al., 2008).

A indústria de carnes está em uma busca constante por soluções para a extensão da vida útil de produtos frescos. A embalagem com atmosfera modificada (ATM) tem sido uma das aplicações propostas mais eficazes para o prolongamento da vida útil de carnes, sendo que seu efeito é baseado na atividade antimicrobiana do dióxido de carbono $\left(\mathrm{CO}_{2}\right)$. A vida útil pode ser consideravelmente prolongada pela modificação da atmosfera que circunda o produto e diminui a atividade dos microrganismos presentes (KOUTSOUMANIS et al., 2008; DEGIRMENCIOGLU et al., 2012). A utilização de embalagens com atmosfera modificada (ATM) tem se apresentado muito eficiente para aumentar a vida útil de carnes frescas, como carne de frango e produtos de peixe (ROTABAKK et al., 2006).

O tempo de vida útil é um atributo importante para os alimentos, sendo que a perda de qualidade sensorial devido a alterações físico-químicas pode ocorrer por fatores como oxidação lipídica e crescimento de micro-organismos deteriorantes. Do ponto de vista microbiológico, o tempo de vida útil pode ser definido como o tempo em que a população microbiana atinge uma determinada concentração.

Os modelos matemáticos são uma importante ferramenta para avaliar o crescimento microbiano em alimentos. Os modelos de microbiologia preditiva têm sido amplamente utilizados e validados para meios sintéticos, e também podem ser utilizados e validados para alimentos armazenados em condições reais. Para isso, entretanto, é necessário considerar no modelo o efeito das mudanças das variáveis externas sobre o crescimento microbiano, como a variação da temperatura com o tempo, por exemplo. O objetivo dessa avaliação é obter predições do crescimento microbiano e da vida útil de alimentos com maior exatidão (CAYRÉ et al., 2003; ZURERA-COSANO et al., 2006).

Whiting e Buchanan (1993) propuseram uma classificação dos modelos matemáticos utilizados na microbiologia preditiva, onde os modelos são divididos em primários, secundários e terciários. Os modelos primários correspondem a modelos matemáticos que descrevem a mudança do número de microrganismos em função do tempo; os modelos secundários são equações que descrevem como os parâmetros de crescimento dos modelos primários variam com a mudança de um ou mais fatores extrínsecos ou intrínsecos, como temperatura, $\mathrm{pH}$, atividade de água, entre outros; e os modelos terciários são aplicativos computacionais utilizados para predizer o crescimento microbiano em diferentes condições de armazenamento.

A maior parte dos modelos encontrados na literatura assume que a temperatura se mantém constante durante todo o armazenamento (DEVLIEGHERE et al., 1998; GIANUZZI et al., 1998, MEZAROBA et al., 2014). Entretanto, em situações reais de processamento, transporte e armazenamento, a temperatura dos alimentos não se mantém constante, apresentando variações importantes que afetam a cinética do crescimento microbiano (VAN IMPE et al., 1992).

Diante disso, o objetivo deste estudo foi propor e validar um modelo matemático para predizer o crescimento de BAL e determinar a vida útil de filés de peito de frango resfriados em condições de armazenamento não-isotérmicas, em embalagens a vácuo e com ATM (com uma concentração de gases $50 \%$ de $\mathrm{CO}_{2}$ e $50 \%$ de $\mathrm{N}_{2}$ ).

\section{MATERIAL E MÉTODOS}

\subsection{AMOSTRAS}

As amostras de filé de peito de frango, sem pele, sem osso, pesando em torno de 400 
gramas (200 gramas cada metade) foram retiradas da linha de abate (todas pertencentes ao mesmo lote) de um frigorífico de aves localizado no estado de Santa Catarina, resfriadas a $0{ }^{\circ} \mathrm{C}$, e posteriormente embaladas a vácuo e em atmosfera modificada. As amostras não passaram por tratamentos para eliminação de micro-organismos, sendo que a contaminação natural das amostras com BAL (com contagem inicial, em média, de $2 \times 10^{1} \mathrm{UFC} / \mathrm{g}$ ) foi considerada para a avaliação do crescimento microbiano.

\subsection{EMBALAGEM E ARMAZENAMENTO}

As amostras de filé de peito de frango foram acondicionadas em sacos plásticos e aplicouse os tratamentos de vácuo e ATM $\left(50 \%\right.$ de $\mathrm{CO}_{2}$ e $50 \%$ de $\left.\mathrm{N}_{2}\right)$. Em seguida, as amostras foram estocadas em condições de armazenamento não isotérmico, sendo que foi programado a mudança de temperatura entre 4 e $12^{\circ} \mathrm{C}$ a cada 12 horas. O registro de temperatura das amostras foi realizado a cada 5 minutos com auxílio de um datalogger (Akso, AK 285).

Os sacos plásticos utilizados como embalagem eram filmes multicamada coextrusado, com camada selante à base de polietileno linear de baixa densidade (PELBD), camada externa à base de Nylon e barreira a oxigênio (etileno vinil álccol - EVOH). Os sacos plásticos possuem barreira com baixa permeabilidade aos gases, cuja permeabilidade ao oxigênio é menor que $3 \mathrm{~cm}^{3} /$ $\mathrm{m}^{2}$.dia (a $23^{\circ} \mathrm{C}, 0 \%$ UR) e permeabilidade ao vapor d'água menor que $6 \mathrm{~g} \mathrm{H}_{2} \mathrm{O} / \mathrm{m}^{2}$.dia $\left(\right.$ a $38^{\circ} \mathrm{C}, 90 \%$ UR), com espessura de $125 \mu \mathrm{m}$ e dimensões de $200 \mathrm{~mm}$ de largura por $260 \mathrm{~mm}$ de comprimento.

O equipamento utilizado para a embalagem foi a embaladora Microvac CV08, marca Selovac. O equipamento permite embalar a vácuo e com ATM, pois o funcionamento do sistema é por substituição mecânica do ar (técnica do vácuo compensado). O cilindro com a mistura gasosa para a embalagem com ATM $\left(50 \%\right.$ de $\mathrm{CO}_{2}$ e $50 \%$ de $\mathrm{N}_{2}$ ) foi cedido pela empresa Linde Gases Ltda.

\subsection{ANÁLISES MICROBIOLÓGICAS}

Amostras de filé de peito de frango foram retiradas do armazenamento não isotérmico a cada dia e 25 gramas da amostra (balança digital Marte, modelo AS 5000) foram coletadas em câmara de fluxo laminar (Valiclean, modelo CL II) e colocadas em saco plástico estéril, seguido de homogeneização em solução salina peptonada $(0,85 \% \mathrm{NaCl} ; 0,1 \%$ peptona) durante 1 minuto em Stomacher (Intersciense, modelo Bag Mixer). Em seguida, as diluições adequadas foram preparadas em duplicata e semeadas em placas de Petri descartáveis estéreis em meio de cultura MRS (Man, Rogosa e Sharpe, marca Merck) em dupla camada para contagem de bactérias lácticas. As placas semeadas foram incubadas em incubadora biológica (Biopar modelo150L) à temperatura de incubação $30^{\circ} \mathrm{C} \pm 1{ }^{\circ} \mathrm{C}$, com tempo de incubação de 48 horas. Após o período de incubação, realizou-se a contagem de unidades formadoras de colônia (UFC) presentes nas placas. Para tanto, utilizou-se o contador de colônias tipo Quebec. O resultado foi expresso em unidades formadoras de colônia por grama de amostra (UFC/g).

\subsection{MODELAGEM MATEMÁTICA}

Os modelos matemáticos de Gompertz modificado (ZWIETERING et al., 1990), Equação (1), e Baranyi (BARANYI E ROBERTS, 1994), Equações (2) e (3), foram utilizados para predizer a cinética de crescimento das BAL em filés de peito de frango armazenados em condições não isotérmicas embalados a vácuo e com ATM. Nas Equações (1), (2) e (3), y(t) é logaritmo neperiano da concentração microbiana (In UFC/g) no tempo t (dias); $y_{0}$ e $y_{\max }$ são o logaritmo neperiano da concentração microbiana inicial e máxima (In UFC/g), respectivamente; $\mu$ é a velocidade específica máxima de crescimento (dias $\left.{ }^{-1}\right) ; \mathrm{Q}(\mathrm{t})$ é uma função do modelo de Baranyi relacionada com o estado fisiológico das células. A condição inicial para resolução 
da Equação diferencial (1) é apresentada na Equação (4), enquanto que as condições iniciais para resolução das Equações diferenciais (2) e (3) são apresentadas nas Equações (5) e (6), respectivamente. A condição inicial $y_{c i}$ bar pode ser obtida diretamente dos dados de crescimento das BAL em condições não isotérmicas, enquanto que as condições iniciais $\mathrm{y}_{\mathrm{ci}}$ gom e $Q_{\mathrm{ci}}$ foram obtidas dos ajustes dos modelos aos dados em condições isotérmicas (MEZAROBA et al., 2014).

$$
\begin{aligned}
& d y(t) / d t=\mu\left[\exp (1) /\left(y_{\max }-y_{0}\right)\right] \ln \left[\left(y_{\max }-y_{0}\right) / y(t)\right] y(t) \\
& d y(t) / d t=\mu\{1 /[1+\exp (-Q(t))]\}\left\{1-\exp \left[y(t)-y_{\max }\right]\right\} \\
& d Q / d t=\mu \\
& y_{\text {ci gom }}=\left(y_{\max }-y_{0}\right) \exp \left\{-\exp \left[\left(\exp (1) \mu \lambda /\left(y_{\max }-y_{0}\right)\right)+1\right]\right. \\
& y_{c i}=y_{0} \\
& Q_{c i}=Q_{0}
\end{aligned}
$$

O parâmetro $\mu$ das equações diferenciais é função da temperatura e os modelos secundários descrevem sua dependência com a temperatura. Os modelos secundários de $\mu$ para o crescimento das BAL em filés de peito de frango embalados a vácuo e com ATM para os modelos de Gompertz modificado ( $\mu_{\mathrm{vac}}$ gom e $\mu_{\mathrm{atm} \text { gom }}$ ) e Baranyi $\left(\mu_{\mathrm{vac}}\right.$ bar e $\mu_{\mathrm{atm} \text { bar }}$ ) foram obtidos por MEZAROBA et al. (2014) e são apresentados nas Equações (7), (8), (9) e (10).

$$
\begin{aligned}
& \mu_{\text {atm gom }}=0,669 \exp (0,103 T) \\
& \mu_{\text {vac gom }}=0,997 \exp (0,090 T) \\
& \mu_{\text {atm bar }}=0,597 \exp (0,136 T) \\
& \mu_{\text {vac bar }}=0,303 \exp (0,158 T)
\end{aligned}
$$

O tempo de vida útil dos filés de peito de frango foi definido neste trabalho como o tempo em que a população microbiana atingiu a concentração de $10^{3} \mathrm{UFC} / \mathrm{g}$, sendo que esse valor foi definido pela observação das alterações organolépticas do produto, quando a contagem de BAL atingiu este valor.

Toda a modelagem matemática foi realizada com o software Matlab R2010a (Natick, USA).

\subsection{ANÁLISES ESTATÍSTICAS}

Os índices estatísticos coeficiente de correlação $\left(R^{2}\right)$, erro médio quadrático (MSE), e os fatores bias e exatidão (ROSS, 1996) foram utilizados para avaliar a qualidade dos modelos matemáticos.

\section{RESULTADOS E DISCUSSÃO}

Os resultados obtidos nos experimentos em condições não isotérmicas são apresentados nas Figuras 1 e 2, as quais contém os perfis de temperatura, os dados experimentais e as predições do crescimento de BAL em filés de peito de frango embalados com ATM (Figura 1) e a vácuo (Figura 2) pelos modelos de Gompertz modificado e Baranyi.

Ao analisar os dados experimentais (círculos) das Figuras 1 e 2 é possível observar que as BAL apresentaram menor crescimento no início do armazenamento em filés de peito de frango embalados com ATM, onde as BAL foram capazes crescer apenas após 4 dias de armazenamento, enquanto que em filés de peito de frango embalados a vácuo, as BAL apresentaram crescimento após 2 dias de armazenamento. 


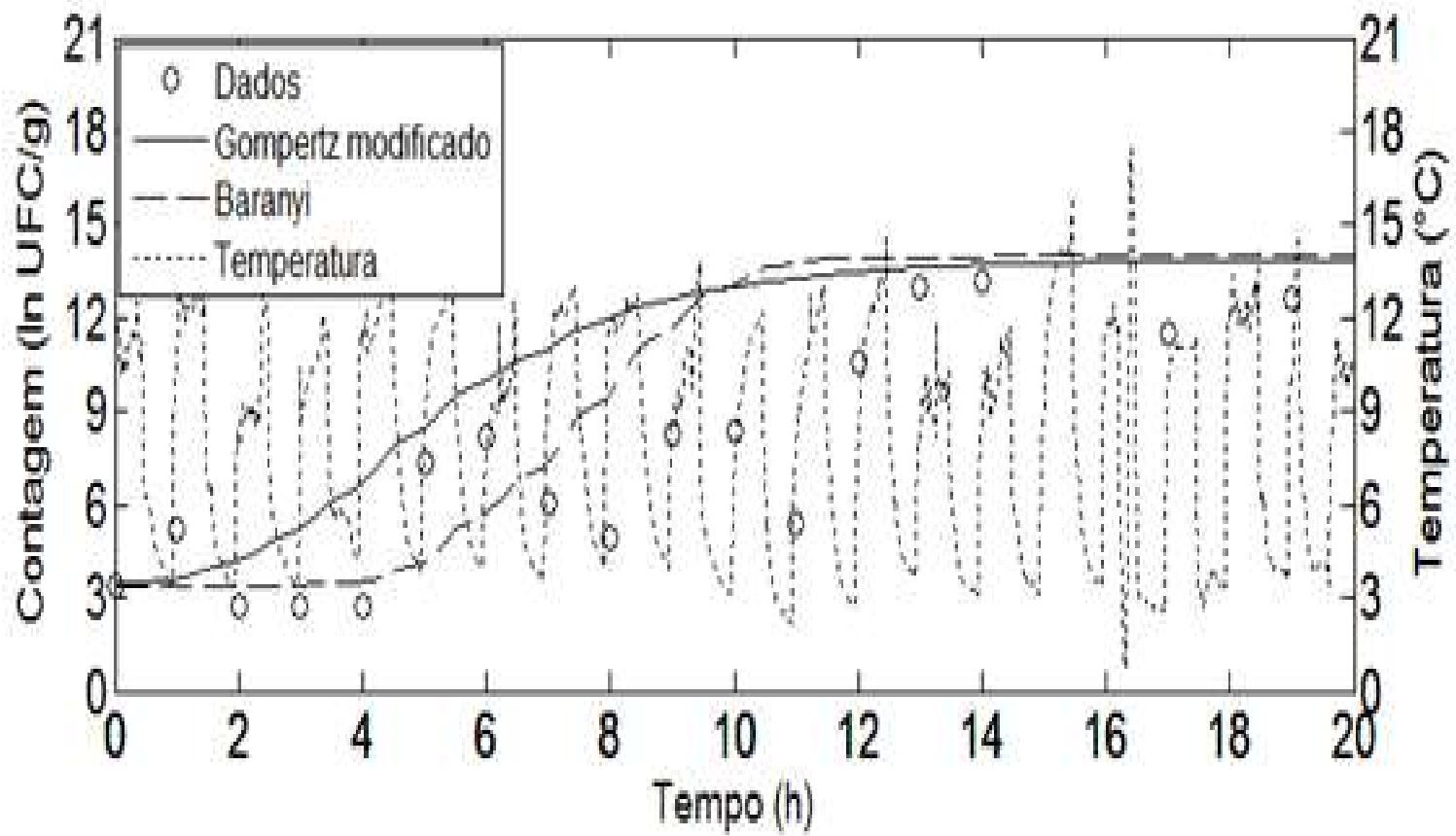

FIGURA 1 - DADOS EXPERIMENTAIS (CÍRCULOS) E PREDIÇÕES DO CRESCIMENTO DE BAL EM FILÉS DE PEITO DE FRANGO EMBALADOS COM ATM PELOS MODELOS DE GOMPERTZ MODIFICADO (LINHA CONTÍNUA) E BARANYI (LINHA TRACEJADA). O PERFIL DE TEMPERATURA DO ARMAZENAMENTO NÃO ISOTÉRMICO É INDICADO PELA LINHA PONTILHADA.

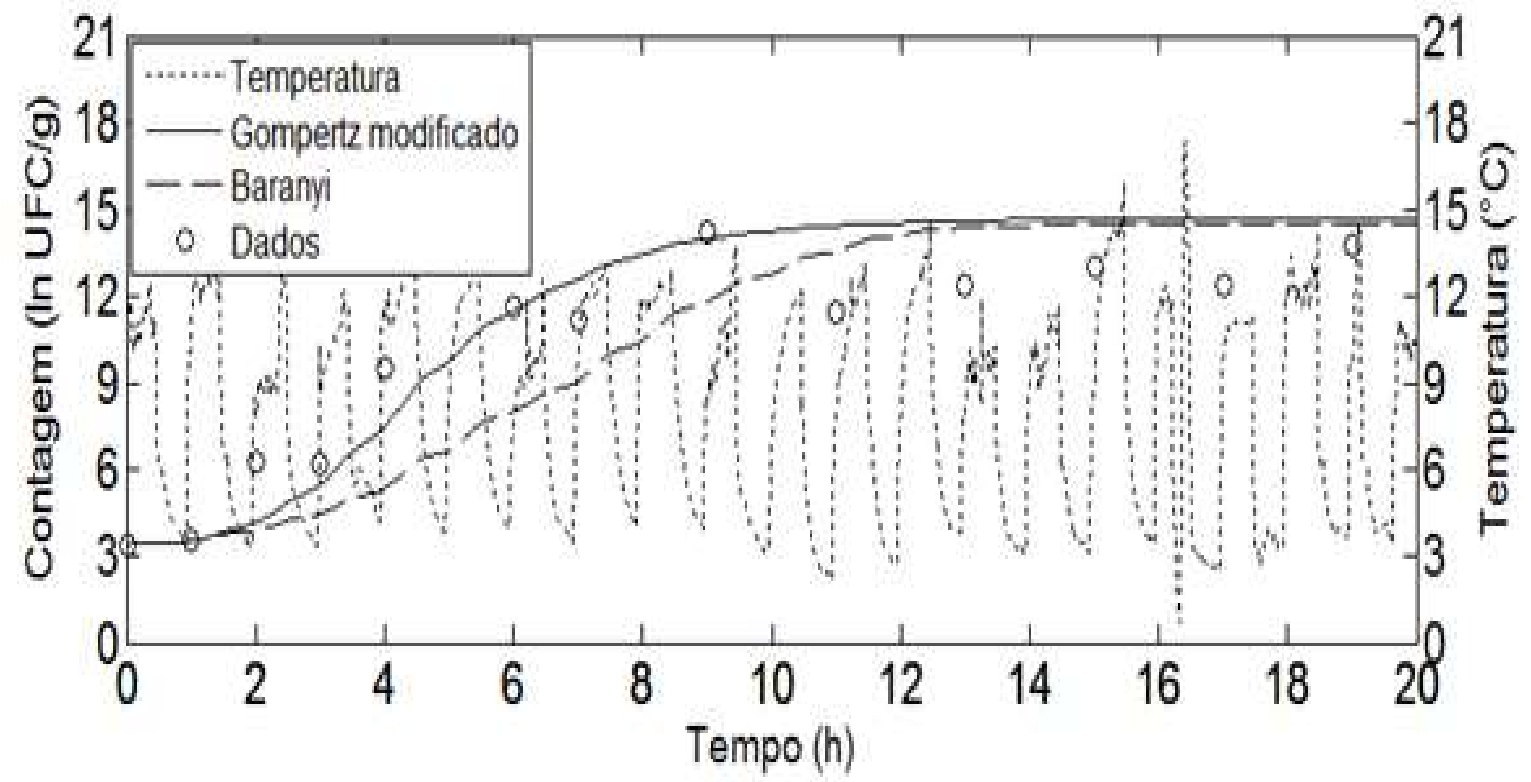

FIGURA 2 - DADOS EXPERIMENTAIS (CÍRCULOS) E PREDIÇÕES DO CRESCIMENTO DE BAL EM FILÉS DE PEITO DE FRANGO EMBALADOS A VÁCUO PELOS MODELOS DE GOMPERTZ MODIFICADO (LINHA CONTÍNUA) E BARANYI (LINHA TRACEJADA). O PERFIL DE TEMPERATURA DO ARMAZENAMENTO NÃO ISOTÉRMICO É INDICADO PELA LINHA PONTILHADA. 
O resultado da análise estatística da qualidade das predições pelos modelos matemáticos de Gompertz modificado e Baranyi em embalagem com ATM e a vácuo em relação aos dados experimentais são apresentados na Tabela 1.

\section{TABELA 1 - VALORES DOS ÍNDICES ESTATÍSTICOS CALCULADOS ENTRE OS VALORES PREDITOS E OBSERVADOS PARA COMPARAÇÃO DA PREDIÇÃO DO CRESCIMENTO DAS BAL EM FILÉS DE PEITO DE FRANGO EMBALADOS EM ATM E A VÁCUO UTILIZANDO-SE OS MODELOS DE GOMPERTZ MODIFICADO E BARANYI NOS TRATAMENTOS NÃO ISOTÉRMICOS PROPOSTOS.}

\begin{tabular}{cccccc}
\hline Embalagem & Modelo & $\mathbf{R}^{2}$ & MSE & Bias & Exatidão \\
\hline \multirow{2}{*}{ ATM } & $\begin{array}{c}\text { Gompertz } \\
\text { modificado }\end{array}$ & 0,603 & 3,479 & 1,431 & 1,498 \\
& Baranyi & 0,620 & 2,607 & 1,173 & 1,388 \\
Vácuo & $\begin{array}{c}\text { Gompertz } \\
\text { modificado }\end{array}$ & 0,830 & 0,939 & 0,979 & 1,156 \\
& Baranyi & 0,645 & 2,168 & 0,850 & 1,286 \\
\hline
\end{tabular}

Ao analisar os resultados da Tabela 1, é possível verificar que ambos os modelos apresentaram predições seguras (fator bias maior que 1) para o crescimento das BAL em filés de peito de frango embalados com ATM, ou seja, o crescimento microbiano predito pelos modelos foi maior que o observado no experimento, e o erro de predição é considerado um erro seguro (Ross, 1996). Por outro lado, ambos os modelos apresentaram predições perigosas (fator bias menor que 1) para o crescimento das BAL em filés de peito de frango embalados a vácuo, onde o crescimento microbiano predito pelos modelos foi menor que o observado no experimento, e o erro de predição é considerado um erro perigoso.

Os erros de predição do crescimento microbiano pelos modelos matemáticos podem ocorrer por diversas razões, sendo que algumas delas são a qualidade dos modelos secundários e o perfil de temperatura não isotérmico avaliado. Juneja et al. (2007) chegaram a conclusão que o desempenho satisfatório de predição de um modelo matemático para o crescimento microbiano em condições não isotérmicas depende do desempenho dos modelos primários e secundários em condições isotérmicas. Entretanto, Longhi et al. (2013) verificaram que a capacidade preditiva dos modelos matemáticos é menor quando ocorrem variações bruscas de temperatura durante o armazenamento, o que pode ser verificado nos perfis de temperatura obtidos no presente trabalho. Dalcanton (2010) também obteve falhas seguras de predição do crescimento de Lactobacillus plantarum em meio MRS em três condições não isotérmicas com variações bruscas de temperatura usando o modelo de Gompertz modificado.

Ao analisar de forma comparativa o desempenho de predição dos modelos de Gompertz modificado e Baranyi através dos índices estatísticos R2, MSE e fator exatidão, é possível verificar que o modelo de Baranyi apresentou melhor predição para o crescimento das BAL em filés de peito de frango embalados com ATM, enquanto que o modelo de Gompertz apresentou melhor predição para as amostras embaladas a vácuo.

De forma comparativa, é possível verificar que o modelo de Gompertz modificado apresentou predições do crescimento das BAL mais conservadoras para os filés de peito de frango embalados tanto com ATM como a vácuo, ou seja, o crescimento das BAL predito pelo modelo de Gompertz é mais rápido em relação ao modelo de Baranyi. Por consequência, esse comportamento 
refletiu na predição da duração da vida útil dos filés de peito de frango, como pode ser observado na Tabela 2, onde a vida útil dos filés de peito de frango predita pelo modelo de Gompertz modificado é menor que a predita pelo modelo de Baranyi, tanto para embalagem com ATM como a vácuo.

\section{TABELA 2 - VALORES DA VIDA ÚTIL OBSERVADA E PREDITA PELOS MODELOS MATEMÁTICOS DE GOMPERTZ MODIFICADO E BARANYI PARA O CRESCIMENTO DE BAL EM FILÉS DE PEITO DE FRANGO EMBALADOS COM ATM E A VÁCUO.}

\begin{tabular}{cccc} 
& & \multicolumn{2}{c}{ Vida útil predita } \\
Embalagem & Vida útil observada* & Gompertz modificado & Baranyi \\
\cline { 3 - 4 } & 12 dias & 7,4 dias & 8,9 dias \\
ATM & 6 dias & 6,0 dias & 8,5 dias \\
\hline
\end{tabular}

*Valores aproximados baseados na observação dos dados experimentais.

Os modelos matemáticos propostos neste estudo podem ser utilizados para verificar a influência que a variação da temperatura de armazenamento tem sobre o crescimento das BAL em filés de peito de frango.

\section{CONCLUSÃO}

Os modelos de Gompertz modificado e Baranyi avaliados neste estudo apresentaram boa capacidade para predizer o crescimento das BAL em filés de peito de frango embalados a vácuo e com ATM em condições não isotérmicas de armazenamento. De forma complementar, foi possível observar que o modelo de Gompertz modificado apresentou predições mais conservadoras sobre a duração da vida útil do produto. Os modelos matemáticos propostos foram validados com dados experimentais obtidos em condições não isotérmicas com mudanças abruptas na temperatura, sendo que essas mudanças abruptas provocaram pequenas falhas de predição pelos modelos.

\footnotetext{
ABSTRACT

PREDICTING SHELF LIFE OF COLD CHICKEN BREAST FILLETS PACKED UNDER VACUUM AND MODIFIED ATMOSPHERE STORED IN NON-ISOTHERMAL CONDITIONS

Temperature is one environmental factor that have great impact on growth of spoilage microorganisms in foods. In real conditions of storage, transportation and commercialization of meat products great flutuation in temperature can occur, and therefore, the study of the growth of lactic acid bacteria (LAB) in non-isothermal conditions has great importance. The aim of this study was to develop and validate mathematical models (modified Gompertz and Baranyi) to non isothermal conditions and to predict the shelf life of cold chicken breast fillet from the growth of LAB in vacuum and modified atmosphere packaging (MAP, $50 \% \mathrm{CO}_{2}$ and $50 \% \mathrm{~N}_{2}$ ). The results indicate that the modified Gompertz and Baranyi models were able to predict the growth of BAL in chicken breast fillets under vacuum and MAP, which was verified through statistical indices $R^{2}$, MSE, bias and accuracy
} 
factors, in which the modified Gompertz model had the most conservative predictions (lower values) for the shelf life duration. The observed shelf life of chicken breast fillets was approximately 12 and 6 days on packages with MAP and vacuum, respectively, showing that the MAP led to increase the shelf life of the product. Moreover, the mathematical models proposed and validated in this study can be used to predict the influence of different non isothermal conditions on the growth of LAB in chicken breast fillets.

\section{KEY-WORDS: LACTIC ACID BACTERIA; MICROBIAL GROWTH IN FOOD; PREDICTIVE MICROBIO-} LOGY.

\section{REFERÊNCIAS}

1 BARANYI, J., ROBERTS, T.A. A dynamic approach to predicting bacterial growth in food. International Journal of Food Microbiology, v.23, p.277-294, 1994.

2 BORCH, E.; KANT-MUEMANSB, M. L.; BLIXT, Y. Bacterial spoilage of meat products and cured meat. International Journal of Food Microbiology, v. 33, p. 103-120, 1996.

3 CAYRÉ, M. E.; VIGNOLO, G.; GARRO, O. Modeling lactic acid bacteria growth in vacuum-packaged cooked meat emulsions stored at three temperatures. Food Microbiology, v. 20, p. 561-566, 2003.

4 DALCANTON, F. Modelagem matemática do crescimento de bactérias ácido lácticas em condições isotérmicas e não isotérmicas. Tese de Doutorado. Florianópolis. 2010.

5 DEVLIEGHERE, F.; DEBEVERE, J.; VAN IMPE, J. Concentration of predict carbon dioxide in the water-phase as a parameter to model the effect of a modified atmosphere on microrganisms. International Journal of Food Microbiology, v. 43, p.105-113, 1998.

6 DEGIRMENCIOGLU, N.; ESNER, O. K.; IRKIN. R.; DEGIRMENCIOGLU, A. Effects of Vacuum and Modified Atmosphere packaging on shelf life extention of minced meat chemical and microbiological changes. Journal of animal and Veterinary Advances, v. 11, p. 898-911, 2012.

7 GIANNUZZI, L., PINOTTI, A., ZARITZKY, N. Mathematical modeling of microbial growth in packaged refrigerated beef at different temperature. International Journal of Food Microbiology, v.39, p.101-110, 1998.

8 JUNEJA, V.K.; MELENDRES, M.V.; HUANG, L.; GUMUDAVELLI V.; SUBBIAH J. THIPPAREDDI H. Modeling the effect of temperature on growth of Salmonella in chicken Food Microbiology, v. 24, p. 328-335, 2007.

9 KOUTSOUMANIS, K. P.; STAMATIOU, A. P.; DROSINOS, E. H.; NYCHAS, G. J. E. Control of spoilage microorganisms in minced pork by a self-developed modified atmosphere induced by the respiratory activity of meat microflora. Food Microbiology, v. 25, p. 915-921, 2008.

10 LONGHI D.A.; DALCANTON, F.; ARAGÃO, G.M.F. DE; CARCIOFI, B A.M., LAURINDO, J.B. Assessing the predictionability of different mathematical models for the growth of Lactobacillus plantarum under non-isothermal conditions. Journal of Theoretical Biology. v. 335, p. 88-96, 2013.

11 MEZAROBA, M.E. DE P.C.; LONGHI, D.A.; VEDOVATTO, E.; ARAGÃO, G.M.F. Estimativa da vida útil e dos parâmetros de crescimento de bactérias ácido-lácticas em filés de peito de frango resdfriados embalados a vácuo e com atmosfera modificada. 2014

12 NYCHAS, G. J.; SKANDAMIS, P. N.; TASSOU, C. C.; KOUTSOUMANIS, K. P.. Meat spoilage during distribution. Meat Science, v. 78, 77-89, 2008.

13 ROSS, T. Indices for performance evaluation of predictive models in food microbiology. Journal of Applied Bacteriology 81, 501-508.1996.

14 ROTABAKK, B. T.; BIRKELAND, S.; JEKSRUD, W. K.; SIVERTSVIK, M. Effect of Modified Atmosphere packaging and Soluble Gas Stabilization on the Shelf Life of Skinless Chicken Breast Fillets. Journal of Food Science, v. 71, p. S124S131, 2006.

15 VAN IMPE, J. F.; BART, I.; NICOLAI, B. M.; MARTENS, T, BAERDEMAEKER, J.; VANDEWALLE J. Dynamic Mathematical Model To Predict Microbial Growth and Inactivation during Food Processing, Applied And Environmental Microbiology, v. 58(9), p. 2901-2909, 1992.

16 WHITING, R. C.; BUCHANAN, R. L. A. Classification of Models for Predictive Microbiology. Food Microbiology, v.10, p. 175-177, 1993.

17 ZURERA-COSANO, G.; GARCÍA-GIMENO, R.M.; RODRÍGUEZ-PÉREZ, R.; HERVÁS-MARTÍNEZ, C. Performance of response surface model for prediction of Leuconostoc mesenteroides growth parameters under different experimental conditions. Food Control, v. 17(6), p. 429-438, 2006.

18 ZWIETERING, M.H., JONGEBURGER, I., ROMBOUTS, F.M., RIET, K.V. Modeling of bacterial growth curve. Applied and Environmental Microbiology, v.56, p.1875 1881, 1990. 